\title{
Research on the Cyclical Fluctuation of Urban Real Estate in China Jiang CHANG*, Guo-tie CHEN
}

\author{
School of Management, Fujian University of Technology, Fuzhou, China
}

${ }^{*}$ Corresponding author

\begin{abstract}
Keywords: Periodic Fluctuation; Diffusion Index; Principal Component Analysis.
\end{abstract}
\begin{abstract}
The impact of the real estate industry on the world economy is unprecedented. Just as the institutional changes in the real estate and mortgage markets in the United States have already had a significant impact on the conduction of monetary policy and the vitality of the economic cycle. As the world's second largest economy, China's real estate market's impact on the global economy cannot be ignored. However, the uniqueness of the Chinese real estate market and the weakness of the data base have greatly increased the difficulty of real estate cycle research. The existing research literature on the cyclical changes in China's real estate market is mostly at the macro level. Based on the concept and theory, this paper analyzed the characteristics of cyclical fluctuations in the real estate market and its influencing factors. Combined with the official data of China National Bureau of Statistics and Local Bureau of Statistics, the authors analyzed the real estate cycle of a metropolitan area in southeastern China by using the composite index composed of diffusion index and principal component analysis, which provides research ideas for real estate development and regional economic development research, and provides the basis for the development of macro-control policies.
\end{abstract}

\section{Background}

Since 1998, China's macroeconomic performance has been markedly deflationary, the overall price level continues to decline, while the real estate market is booming. On the real estate bubble, overheated investment and other issues have been debated, and the real estate industry has become a hot topic of various studies. Although real estate has immovable features, the real estate market in different countries and cities is quite different [1]. Therefore, it is of practical significance to analyze the periodicity of regional real estate market with economic, political and cultural characteristics.

\section{The Real Estate Cycle Fluctuations Theory and Measure Method}

Major advances in macroeconomic monitoring and early warning systems were made in the 1950s. In 1950, Moore, an economic statistician at the National Institute of Economic Research (NBER), established a new climate monitoring system based on the monitoring indicator system in the 1930s [2]. It consists of leading index, synchronization index and lagging index. Taking the macroeconomic comprehensive status as the measuring object, a new multi-index information synthesis method - diffusion index method (DI) is adopted. However, DI can not accurately reflect the results of more specific factors, as comprehensive assessment tends to inhibit the role of sensitive factors, while the effect on non-sensitive factors has been enhanced, so the interference is relatively large.

Hirschman, the chief economic statistician of the Bureau of Economic Analysis of the United States Department of Commerce, proposed the Composite Index (CI) in the 1960s to synthesize multi-index information. It used techniques such as multiple standardization of indicators, correction of symmetry, first-order difference and trend adjustment to deal with various indicators so as to reflect the impact of each indicator on the economic operation and reflect the fluctuation of the economy and thus make up for the deficiency of the diffusion index DI. Therefore, the composite index CI has a tremendous impact on the economic monitoring and early warning system both from theory and practice, and has also become one of the basic theories and basic methods for 
monitoring and early warning systems.

In this paper, we used the composite index composed of diffusion index and principal component analysis to quantitatively analyze the real estate cycle, form the cycle fluctuation graph, analyze the main factors that affect the real estate cycle fluctuation, and predict the real estate cycle trend.

\section{Fuzhou Real Estate Cycle Fluctuations in Empirical Analysis}

\subsection{Fuzhou real estate market cycle fluctuation index system}

In order to objectively analyze the real estate running track, correctly evaluate the real estate situation, the preparation of the real estate market index system is necessary. Its design principles are comprehensiveness, simplicity and maneuverability. The data set of this paper includes data on various parameters of the real estate industry in metropolitan areas in southeastern China from 2002 to 2015, as well as data on economic factors affecting cyclical real estate fluctuations. The data comes from the official website of the Bureau of Statistics of China, the Statistics Bureau of Fujian Province and the Bureau of Statistics of Fuzhou City.

According to the analysis of factors affecting the cyclical fluctuations and design principles, 10 indicators were selected:

Table 1. Real estate influencing factors

\begin{tabular}{ll}
\hline Independent variable & Index name (\%) \\
\hline X1 & Annual growth rate of GDP \\
X2 & Annual growth rate of fixed asset investment \\
X3 & Annual growth rate of investment in real estate development \\
X4 & Annual growth rate of purchased land area \\
X5 & Annual growth rate of land area under construction \\
X6 & Annual growth rate of completed housing area \\
X7 & Per capita annual income growth rate \\
X8 & Annual growth rate of housing sales \\
X9 & Annual growth rate of housing sales area \\
X10 & Annual growth rate of average sales price of housing \\
\hline
\end{tabular}

\subsection{Fuzhou real estate market cycle fluctuation diffusion index}

The diffusion index at the inflection point of the overall economic cycle should be $50 \%$. That is, if the diffusion index value between $0 \%-50 \%$, the economy is in the contraction phase; and when the value between $50 \%-100 \%$, the economy is in the expansion phase. According to the definition of diffusion index, the dynamic spread index of Fuzhou real estate market growth is calculated. The specific value is shown in Table 2.

Table 2. Fuzhou real estate market growth volatility cycle spread index

\begin{tabular}{lllllllllllll}
\hline Year & $\mathbf{2 0 0 4}$ & $\mathbf{2 0 0 5}$ & $\mathbf{2 0 0 6}$ & $\mathbf{2 0 0 7}$ & $\mathbf{2 0 0 8}$ & $\mathbf{2 0 0 9}$ & $\mathbf{2 0 1 0}$ & $\mathbf{2 0 1 1}$ & $\mathbf{2 0 1 2}$ & $\mathbf{2 0 1 3}$ & $\mathbf{2 0 1 4}$ & $\mathbf{2 0 1 5}$ \\
\hline Diffusio & 30.00 & 30.00 & 70.00 & 70.00 & 0.00 & 80.00 & 60.00 & 70.00 & 30.00 & 60.00 & 0.00 & 60.00 \\
n index & $\%$ & $\%$ & $\%$ & $\%$ & $\%$ & $\%$ & $\%$ & $\%$ & $\%$ & $\%$ & $\%$ & $\%$ \\
\hline
\end{tabular}

\section{Fuzhou Real Estate Cycle Comprehensive Index Calculation}

\subsection{Inspection}

The main requirement of principal component analysis is that the correlation between the variables is high. Each index is calculated by SPSS software have a high correlation coefficient, And by KMO and Bartlett's test, the description can be analyzed using the principal component analysis method. 


\subsection{Select the main component}

According to the eigenvalues and contribution rates calculated by SPSS, consider two criteria can determine the two principal components. The cumulative contribution rate of the first two major parts has reached 91, indicating that most of the original information can be reflected.

Table 3. Contribution rates of major components

\begin{tabular}{l|l|l|l|l|l|l}
\hline \multirow{2}{*}{$\begin{array}{l}\text { Componen } \\
\mathrm{t}\end{array}$} & \multicolumn{3}{|l|}{ Initial Eigenvalues } & \multicolumn{3}{l|}{ Extraction Sums of Squared Loadings } \\
\cline { 2 - 6 } & Total & \% of Variance & Cumulative \% & Total & \% of Variance & Cumulative \% \\
\hline 1 & 8.145 & 81.454 & 81.454 & 8.145 & 81.454 & 81.454 \\
2 & 1.009 & 10.089 & 91.542 & 1.009 & 10.089 & 91.542 \\
3 & .464 & 4.637 & 96.180 & & & \\
4 & .307 & 3.071 & 99.251 & & & \\
5 & .044 & .442 & 99.693 & & & \\
6 & .014 & .140 & 99.833 & & & \\
7 & .010 & .099 & 99.933 & & & \\
8 & .005 & .054 & 99.986 & & & \\
9 & .001 & .013 & 99.999 & & & \\
10 & $7.502 \mathrm{E}-05$ & .001 & 100.000 & & & \\
\hline
\end{tabular}

\subsection{Synthetic composite index}

According to the principle of principal component analysis method, the contribution rate of each principal component is weighted and the comprehensive index $\mathrm{Y}$ is synthesized, the specific value in Table 4.

Table 4. Principal Component Score and Consolidated Index

\begin{tabular}{lccc}
\hline & Z1 & Z2 & Y \\
\hline 2003 & -3.3450 & 1.2325 & -2.6002 \\
2004 & -3.2061 & 1.7720 & -2.4326 \\
2005 & -2.1435 & -0.0996 & -1.7559 \\
2006 & -2.1799 & 0.1690 & -1.7584 \\
2007 & -1.6314 & -0.8041 & -1.4099 \\
2008 & -1.9225 & -2.0120 & -1.7689 \\
2009 & -0.9767 & -0.3560 & -0.8314 \\
2010 & -0.3725 & -0.5518 & -0.3591 \\
2011 & 0.9219 & 0.0081 & 0.7517 \\
2012 & 2.1496 & -0.7776 & 1.6724 \\
2013 & 4.0798 & 1.2806 & 3.4522 \\
2014 & 4.0293 & -0.0485 & 3.2769 \\
2015 & 4.5971 & 0.1872 & 3.7632 \\
\hline
\end{tabular}

\section{Comprehensive Analysis on the Fluctuation of Real Estate Market Growth in Fuzhou}

The comparison between the diffusion index and the comprehensive index analysis shows that the analysis of Fuzhou real estate growth cycle is basically the same (Fig. 1). However, there are differences in the judgment of the cycle in 2012, mainly because the calculation of the diffusion index is susceptible to minor changes, which was affected by the global financial crisis in 2012, and real estate companies tried to avoid the risk of contraction business, but the overall look was still in the expansion period.

The value added of real estate refers to the final result of the production activities of the real estate enterprises in the form of money in the reporting period. It is a new value in the process of production. It is a comprehensive indicator of real estate. This paper then combines the growth rate of the real estate value-added chain with the composite index to analyze the cycle of Fuzhou real estate market. 


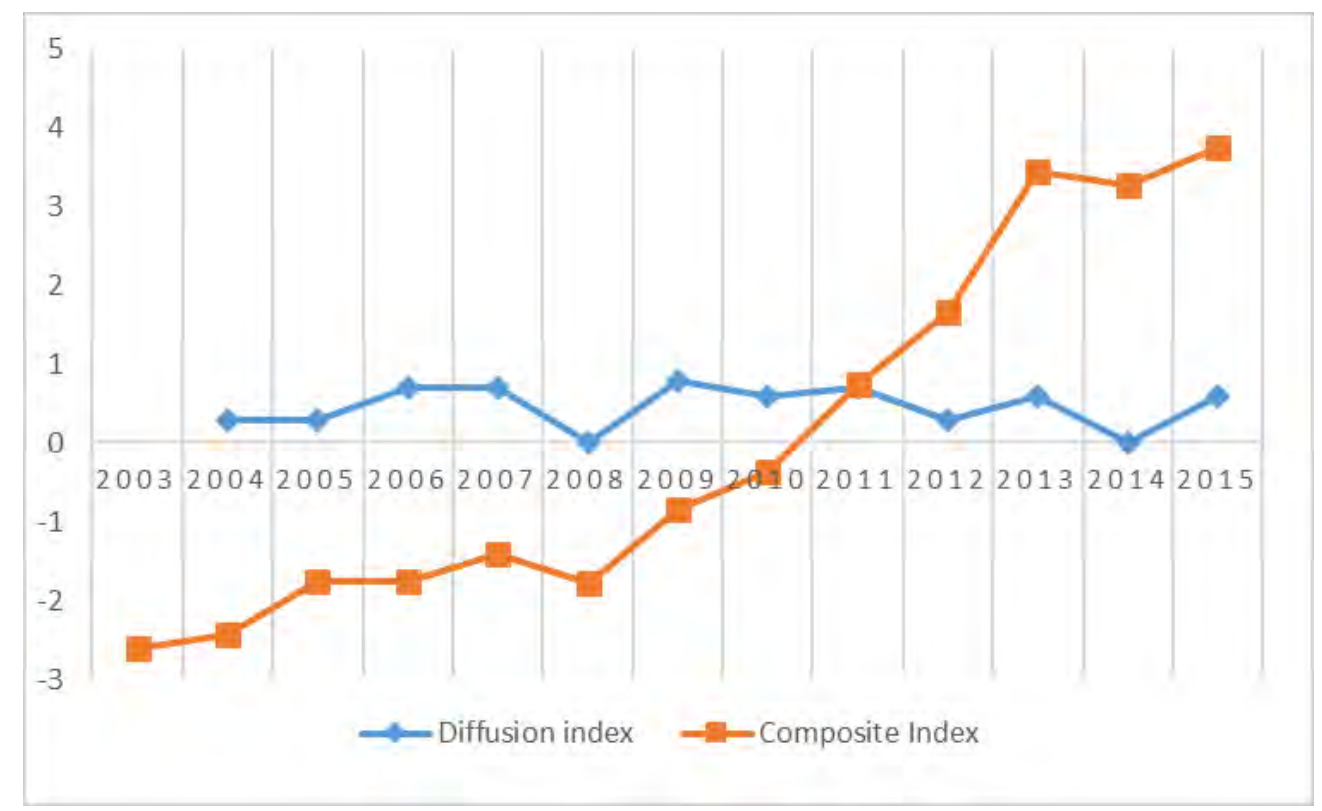

Figure 1. Diffusion index and composite index comparison

As shown in Fig. 2, Fuzhou real estate added value and comprehensive index trend is basically the same, and the range is basically the same. However, there are differences in the judgments of the 2015 cycle, mainly due to the economic downturn, shortage of funds for real estate business, increased inventory pressure, and reduced investment. In 2015, the government introduced a number of real estate destocking policies and increased social capital investment, which was essentially an expansion period. It is shown that the composite index synthesized by principal component method can well reflect the direction and amplitude of periodic fluctuation.

Combining the real estate increase value, diffusion index, and comprehensive index of Fuzhou city, the historical data of the real estate industry in Fuzhou for the past 13 years have been analyzed, and roughly experienced two peaks: one in 2007 and one in 2013 . The two climaxes that have developed since 2003 were mainly caused by the booming supply and demand in the commercial housing market, and by the external environment, especially the fluctuations in the socio-economic environment, and the role of government real estate policies.

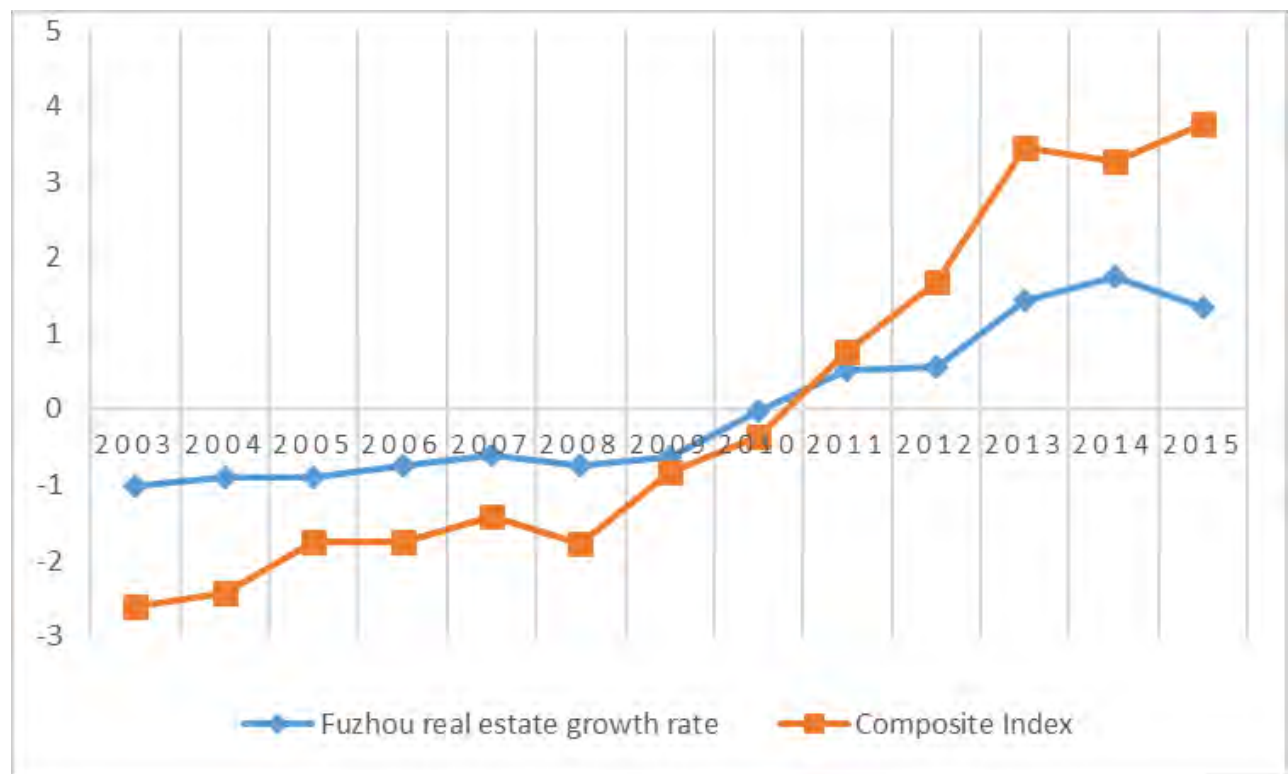

Figure 2. Comparison of Fuzhou Real Estate Growth Rate and Composite Index

\section{Summary}

The establishment of Fuzhou city real estate market cyclical fluctuation index system also has 
shortcomings and difficulties. In this paper, the selection of the above indicators system is not very perfect choice, many important real estate market bases are lack of statistical data, making the selection of indicators is limited. Therefore, we need to learn from foreign and Hong Kong, Taiwan's real estate market statistics system to gradually improve the real estate statistical information system.

\section{Acknowledgement}

This research was financially supported by the Key Project of Fujian Social Science Plan Fund (No. FJ2016A011).

\section{References}

[1] Qian LI. Research on Beijing Real Estate Cycle Fluctuation [D]. Capital University of Economics and Business, 2004. (in Chinese)

[2] Lie-yun Ding, Causes of the real estate cycle fluctuation analysis [J], Huazhong University of Science and Technology (Social Science Edition), 2003, pp. 19-25. (in Chinese) 